\title{
Subepithelial spread of early gastric cancer is common and extends more than expected
}

\author{
Min Kyung Yeo ${ }^{1}$ S Sun Hyung Kang ${ }^{2}$ (I) $\cdot$ Hyuk Soo Eun ${ }^{2} \cdot$ Eaum Seok Lee $^{2} \cdot \mathrm{Hee} \mathrm{Seok} \mathrm{Moon}^{2} \cdot$ Seok Hyun Kim ${ }^{2} \cdot$ \\ Jae Kyu Sung ${ }^{2} \cdot$ Byung Seok Lee $^{2} \cdot$ Hyun Yong Jeong ${ }^{2} \cdot$ Go Eun Bae ${ }^{1}$
}

Received: 16 December 2020 / Accepted: 12 February 2021 / Published online: 23 February 2021

(c) The International Gastric Cancer Association and The Japanese Gastric Cancer Association 2021

\begin{abstract}
Backgrounds The clinical significance of subepithelial (SE) spread from early gastric cancer (EGC) is poorly understood. Thus, we evaluated the proportion and extent of SE spread from EGC, as well as related risk factors.

Methods We reviewed medical records and pathological specimens from patients with EGC who underwent surgery or endoscopic resection between January 2016 and December 2016 at Chungnam National University Hospital.

Results A total of 404 patients were reviewed and SE spread was identified for 142 patients (35.1\%). The presence of SE spread was associated with gender, histological type, location, endoscopic appearance, color change, presence of lymphovascular invasion, and invasion depth. Multivariable analysis revealed that SE spread was only independently associated with histological type. The distance of SE spread was significantly different between histological types, and the maximum distance was $17 \mathrm{~mm}$.

Conclusion More than 30\% of our patients with EGC had SE spread, which could reach up to $17 \mathrm{~mm}$. Given the proportion of SE spread in these cases, a wider resection margin may be safe during endoscopic resection or surgery.
\end{abstract}

Keywords Early gastric cancer $\cdot$ Subepithelial spread $\cdot$ Endoscopic resection

\section{Introduction}

Endoscopic submucosal dissection (ESD) is a major treatment modality for early gastric cancer (EGC), and many studies have evaluated ESD techniques, although there are limited reports regarding a specific margin distance that is needed for safe and effective ESD. Medicine can be considered both an art and a science [1], thus there could be an empirically effective treatments that are not currently supported by high-level evidence. For the safe ESD margin, the only fact we know is that the ESD margin must be sufficiently distanced. Yun et al. [2] have suggested that a margin of $<3 \mathrm{~mm}$ is a risk factor for local recurrence of

Sun Hyung Kang

porrtos@hanmail.net

1 Department of Pathology, School of Medicine, Chungnam National University, Daejeon, Republic of Korea

2 Division of Gastroenterology and Hepatology, Department of Internal Medicine, School of Medicine, Chungnam National University, 282 Munhwa-ro Jung-Gu, Daejeon 35015, Republic of Korea gastric epithelial neoplasia. Empirically, mucosal markings are often placed 3-5 $\mathrm{mm}$ outside the tumor edge and the mucosal incision is made outside these markings [3]. However, we often encounter cases with a shorter safety margin than we expected or a positive lateral margin (LM) after ESD.

Kakushima et al. [4] evaluated possible risk factors for lateral margin (LM) positivity after ESD in cases of EGC, and suggested that LM positivity could be associated with lesions with a flat spreading area, lesions with an unexpected nearby lesion, and lesions with lateral extension beneath the non-cancerous mucosa. Unexpected lateral extension under the normal mucosa is difficult to avoid, while the other two factors can be minimized via better endoscopic image quality and greater endoscopists' experience. Another study evaluated the extent of subepithelial (SE) spread from submucosal invasive EGC, and noted that the maximum extent of SE spreading was $1 \mathrm{~cm}$ in 595 slides of surgically resected specimens from 60 EGC patients [3]. However, that $1-\mathrm{cm}$ margin would be difficult to apply for all patients with EGC, as that study only considered SE spread of submucosal cancer and did not identify the exact proportion of patients with 
SE spread. Our group recently evaluated the proportion of SE spread from early gastric signet ring cell carcinoma [5], although the proportion and extent of SE spread for other histological types are not evaluated. If SE spread is common in EGC, it might be a key factor for determining the resection margin during ESD. Therefore, we aimed to characterize the extent and proportion of SE spread for all histological types of EGC as well as the related risk factors, correlation between SE spread distance and superficial lesion size, and endoscopic/pathological features of SE spread.

\section{Material and methods}

\section{Study population}

We reviewed medical records and pathological specimens from patients with EGC who underwent surgery or endoscopic resection between January 2016 and December 2016 at Chungnam National University Hospital. Sixteen patients with signet ring cell carcinoma were included in this study and were duplicated from our previous study [5]. Cases were excluded if the SE spread or endoscopic images could not be precisely evaluated (e.g., because of missing specimens or poor image quality), or if they involved mixed lesion with cancer and dysplasia (because it would be difficult to define the distal lesion edge). All patients had undergone ESD or subtotal/total gastrectomy with $\mathrm{D} 1+\alpha$ or $\mathrm{D} 2$ lymphadenectomy. All ESD procedures were performed by 4 expert endoscopists who had performed $>5,000$ gastroscopies and $>200$ ESD procedures for EGC. Surgery was performed by two expert surgeons who had performed $>1,000$ gastrectomy.

\section{Pathological evaluation}

All pathological specimens were reviewed by two expert pathologists (MK Yeo and GE Bae). Pathological specimens from gastrectomy were sectioned at 4-mm intervals and pathological specimens from ESD were sectioned at 3-mm intervals. The distance of SE spread was measured during a pathological review as the distance from the edge of the superficial lesion (cancer) to the other edge of the SE spread. The extent of subepithelial spread was measured by calculating the distance from the edge of the cancer-infiltrating epithelium to the other edge of subepithelial spread (Fig. 1). The extent of subepithelial spread was measured under highpower microscopy (X400). The minimum cut-off length was $50 \mu \mathrm{m}$, which was set as the cut-off length for positive subepithelial spread. All resected specimens were reviewed, and the one with longest SE spread was determined as a representative value.
The presence of lymph node (LN) metastasis was only evaluated for patients who underwent surgical resection. The lymphovascular invasion (LVI) status and depth of invasion were evaluated for all subjects. In surgically resected specimens, the submucosal layer was equally divided into three layers (upper third =SM1, middle third = SM2, and lower third=SM3). Among the ESD specimens, those less than $500 \mu \mathrm{m}$ were considered SM1, those that were $500-1000 \mu \mathrm{m}$ were considered SM2, and those above $1000 \mu \mathrm{m}$ were considered SM3.

The histological subtypes were defined as: well differentiated, moderately differentiated, poorly differentiated, mixed type, poorly cohesive carcinoma other than signet ring cell (PCC), pure signet ring cell carcinoma (SRC), mucinous type, and papillary carcinoma. The differentiation between PCC and SRC was based on the recently published consensus guideline [6].

\section{Endoscopic examination}

All endoscopic procedures, including ESD and preoperative gastroscopy, were performed using a video endoscope (H240 and H260; Olympus Medical Systems, Tokyo, Japan), and chromoendoscopy with indigo-carmine was occasionally performed. The endoscopic review was performed by one endoscopist (SH Kang) using images that were saved in the picture archiving and communication system of Chungnam National University Hospital. Endoscopic appearance, color change, and location were evaluated. Endoscopic appearance was classified according to the Paris classification. Color change was defined as redness (patch erythematous change), discoloration (disappearance of the normal mucosa's pinkish color), and mixed pattern (mixture of erythematous change and discoloration). Only the presence of fold convergence was evaluated and we did not consider other detailed fold changes (e.g., abrupt cutting, clubbing, and fusion).

\section{Statistical methods}

All statistical analyses were performed using SPSS software (version 18.0; SPSS Inc., Chicago, IL, USA). Univariate analysis was performed using the Chi-squared test and logistic regression analysis was performed to identify independent risk factors for SE spread. Pearson correlation coefficient was used to evaluate the relationship between superficial lesion size and SE spread distance. The Kruskal-Wallis test was used to evaluate the differences in SE spread distance according to histological types. Results were considered statistically significant at $p$ values of $<0.05$. 


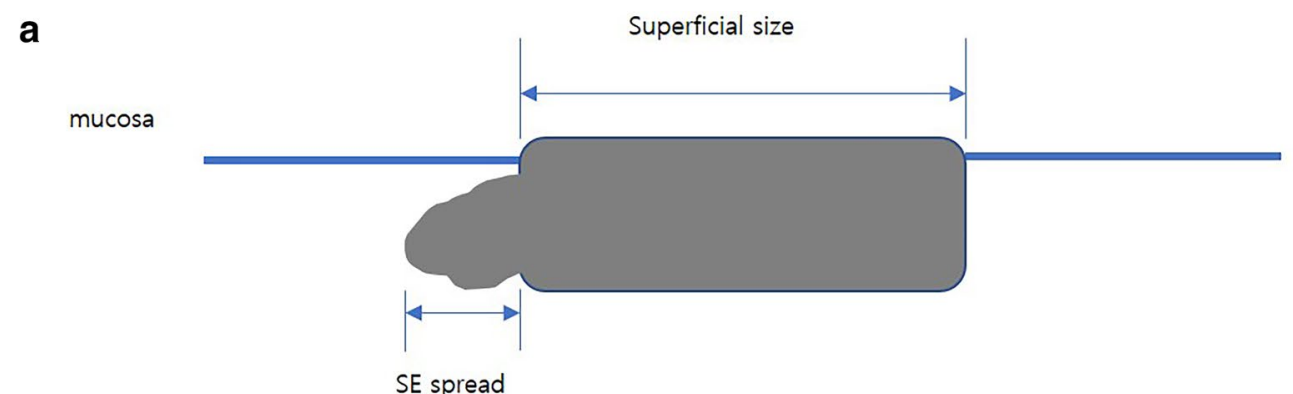

SE spread
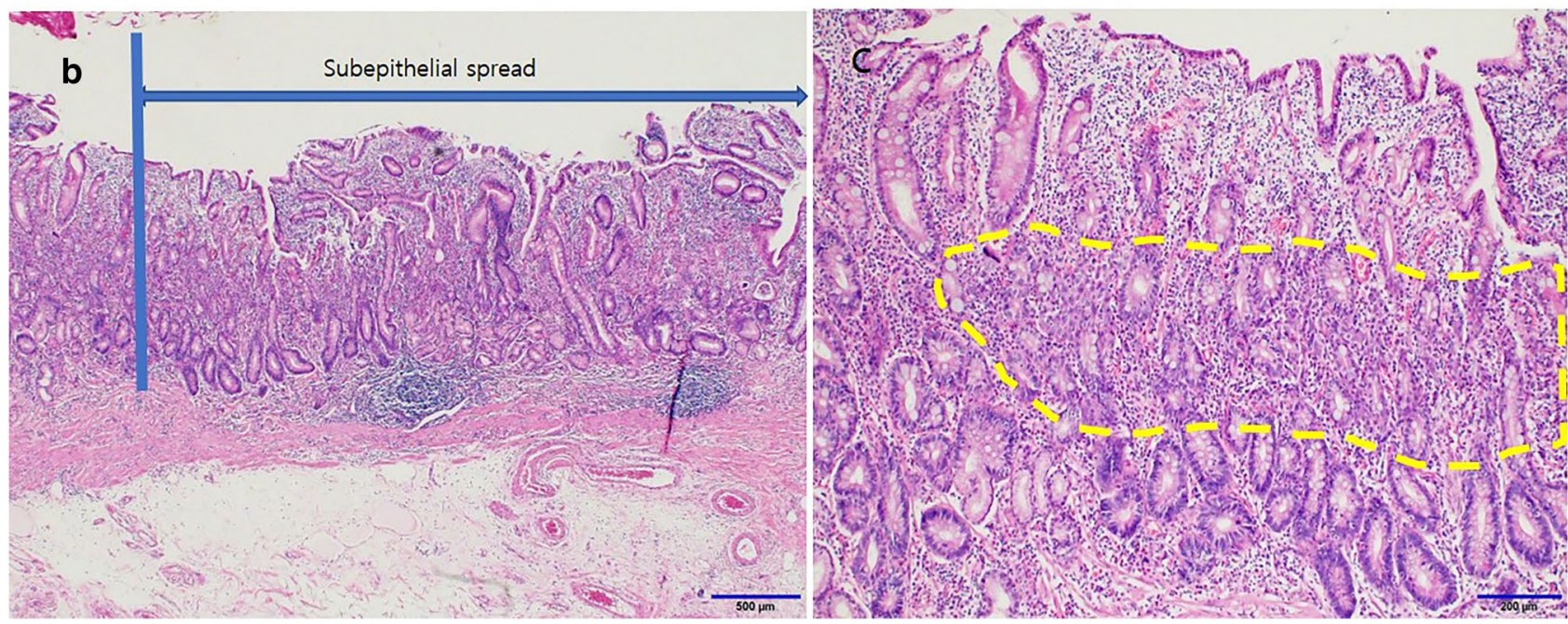

Fig. 1 Subepithelial spreading of early gastric cancer. a Subepithelial (SE) spreading was measured as the distance from the edge of the superficial lesion to the outer edge of the SE spread. b The width of the superficial lesion was defined as the length of cancer-infiltrated epithelium $(\times 40$ image of differentiated tubular adenocarcinoma

\section{Results}

During the study period, 449 patients with EGC underwent surgical resection or ESD at our center, although pathological slides were not available for three patients, as they had been used for other studies. Furthermore, we excluded 14 patients with poor endoscopy image quality or cancer in unexpected locations, and 28 patients with mixed lesion of carcinoma and dysplasia. Thus, the study was performed using data from 236 patients. The mean patient age was 64.83 years, 298 patients $(73.8 \%)$ were men, and 229 patients underwent surgical resection (Table 1).

We identified SE spread in 142 patients (35.1\%), which was significantly associated with gender, histological type, location, endoscopic appearance, color change, presence of LVI, and invasion depth (Table 2). However, SE spread was only associated with histological type in the logistic regression analysis (Table $3, p=0.000$ ). Location, endoscopic appearance, color change, presence of LVI, and spreading in the subepithelial layer, hematoxylin and eosin staining). c The tumor is covered by normal gastric glands, and fused differentiated tumor glands are visible inside the intramucosal layer $(\times 100$, hematoxylin and eosin staining)

Table 1 Demographics

\begin{tabular}{ll}
\hline Characteristics & Number \\
\hline Sex (M:F) & $298: 106$ \\
Endoscopic features & \\
I & 16 \\
IIa & 73 \\
IIb & 59 \\
IIc & 214 \\
III & 42 \\
LN metastasis (Y:N) & $218: 11$ \\
Treatment modality & \\
Surgery & 229 \\
ESD & 175 \\
\hline
\end{tabular}

$M$ male, $F$ female, $L N$ lymph node, $Y$ yes, $N$ no

invasion depth were not significant on logistic regression analysis. 
Table 2 Univariate analysis for presence of SE spread

\begin{tabular}{|c|c|c|c|}
\hline \multirow{2}{*}{$\begin{array}{l}\text { Characteristics } \\
\text { Gender }\end{array}$} & \multicolumn{2}{|c|}{ Presence of SE spread } & \multirow{2}{*}{$\frac{p \text { value }}{\mathbf{0 . 0 0 0}}$} \\
\hline & No & Yes & \\
\hline Male & 209 & 89 & \\
\hline Female & 53 & 53 & \\
\hline Histology & & & 0.000 \\
\hline Well & 81 & 6 & \\
\hline Moderate & 152 & 50 & \\
\hline Poorly & 11 & 19 & \\
\hline Mixed & 6 & 9 & \\
\hline PCC & 7 & 39 & \\
\hline SRC & 3 & 19 & \\
\hline Mucinous & 1 & 0 & \\
\hline Papillary & 1 & 0 & \\
\hline Location & & & 0.001 \\
\hline Cardia & 6 & 3 & \\
\hline Fundus & 0 & 1 & \\
\hline Upper body & 17 & 6 & \\
\hline Mid-body & 21 & 21 & \\
\hline Lower body & 47 & 42 & \\
\hline Angle & 30 & 23 & \\
\hline Antrum & 141 & 46 & \\
\hline Endoscopic appearance & & & 0.002 \\
\hline I & 11 & 5 & \\
\hline IIa & 62 & 11 & \\
\hline IIb & 34 & 25 & \\
\hline IIc & 132 & 82 & \\
\hline III & 23 & 19 & \\
\hline Fold convergence & & & 0.009 \\
\hline No & 223 & 104 & \\
\hline Yes & 39 & 38 & \\
\hline Elevation & & & 0.000 \\
\hline No & 137 & 103 & 0.000 \\
\hline Yes & 125 & 39 & \\
\hline Flat appearance & & & 0.008 \\
\hline No & 220 & 104 & \\
\hline Yes & 42 & 38 & \\
\hline Color change & & & 0.009 \\
\hline Redness & 147 & 66 & \\
\hline Discoloration & 70 & 33 & \\
\hline Mixed & 45 & 43 & \\
\hline LVI & & & 0.000 \\
\hline No & 234 & 107 & \\
\hline Yes & 28 & 35 & \\
\hline Depth & & & 0.004 \\
\hline M & 207 & 95 & \\
\hline SM1 & 25 & 12 & \\
\hline SM2 & 15 & 22 & \\
\hline SM3 & 15 & 13 & \\
\hline
\end{tabular}

$\chi^{2}$ test: endoscopic appearance was classified according to the Paris classification

$S E$ subepithelial spread, $P C C$ poorly cohesive carcinoma other than pure signet ring cell carcinoma, $S R C$ pure signet ring cell carcinoma, $L V I$ lymphovascular invasion, $M$ mucosal cancer, $S M$ submucosal cancer
Table 3 Logistic regression analysis for SE spread in EGC

\begin{tabular}{lrrll}
\hline Characteristics & $\beta$-coefficient & OR & $95 \%$ CI & $p$ value \\
\hline $\begin{array}{l}\text { Histology } \\
\quad \text { ref.=well) }\end{array}$ & & & & 0.000 \\
Moderate & 1.570 & 4.809 & $1.836-12.595$ & 0.001 \\
Poorly & 2.894 & 18.057 & $5.397-60.411$ & 0.000 \\
Mixed & 2.822 & 16.813 & $3.874-72.968$ & 0.000 \\
PCC & 4.007 & 54.982 & $15.651-193.149$ & 0.000 \\
SRC & 4.114 & 61.161 & $12.513-298.947$ & 0.000 \\
mucinous & -19.725 & 0.000 & 0.000 & 1.000 \\
Papillary & -18.348 & 0.000 & 0.000 & 1.000 \\
\hline
\end{tabular}

All variants which are statistically significant in Chi-square were included, and only histology was significant in logistic regression analysis

$S E$ subepithelial spread, ref. reference, $O R$ odds ratio, $C I$ confident interval, $P C C$ poorly cohesive carcinoma other than pure signet ring cell carcinoma, $S R C$ pure signet ring cell carcinoma

Furthermore, the SE spread distance varied significantly according to histological type (Kruskal-Wallis test, $p=0.000$ ), with greater SE spread distances observed for the mixed and PCC types (Table 4; Fig. 2). The maximum SE spread distance was $17 \mathrm{~mm}$, which was observed for mixed type. The SE spread distance was not correlated with the superficial lesion size (Fig. 3, $p=0.056$ ).

Subgroup analysis was performed in patients who had negative submucosal and lymphatic invasion, which revealed that SE spread was significantly associated with gender, histology, location, endoscopic appearance, and color change (Table 5). Similar to among all patients, histologic type was the only significant factor in patients who had negative submucosal and lymphatic invasion in logistic regression analysis (Table 6). Relative to well-differentiated cancers, significantly increased risks of SE spread were observed for moderately differentiated cancers, poorly differentiated cancers, mixed type, PCC, and SRC $(p<0.05)$. The mean SE spread distance was highest in cases of mixed type, and also the largest SE spread distance $(17 \mathrm{~mm})$ was observed in mixed type (Fig. 4, Table 7).

\section{Discussion}

Determining the safe resection margin for ESD is a fundamental issue that has been overlooked. Ironically, this issue was not properly investigated as endoscopists became accustomed to maintaining wide margins with the advances in ESD technology. Extensive resection may increase oncological safety, although wider margins also create longer procedure times and more complications. 
Table 4 Difference of extent of SE spread between histologic types

\begin{tabular}{llllcl}
\hline Histology & Mean $(\mathrm{mm})$ & SD & Minimum $(\mathrm{mm})$ & Maximum $(\mathrm{mm})$ & Number \\
\hline Well & 1.8333 & 1.06905 & 1.00 & 4.00 & 6 \\
Moderate & 2.0200 & 1.63495 & 1.00 & 10.00 & 50 \\
Poorly & 2.6316 & 1.80156 & 1.00 & 7.00 & 19 \\
Mixed & 6.3333 & 5.22015 & 1.00 & 17.00 & 9 \\
PCC & 4.4872 & 2.40473 & 1.00 & 10.00 & 39 \\
SRC & 1.9226 & 2.03966 & 0.05 & 6.00 & 19 \\
Total & 3.0319 & 2.63069 & 0.05 & 17.00 & 142 \\
\hline
\end{tabular}

Kruskal-wallis test: $p$ value $=0.000$

$S E$ subepithelial spread, $S D$ standard deviation, $P C C$ poorly cohesive carcinoma other than pure signet ring cell carcinoma, $S R C$ pure signet ring cell carcinoma

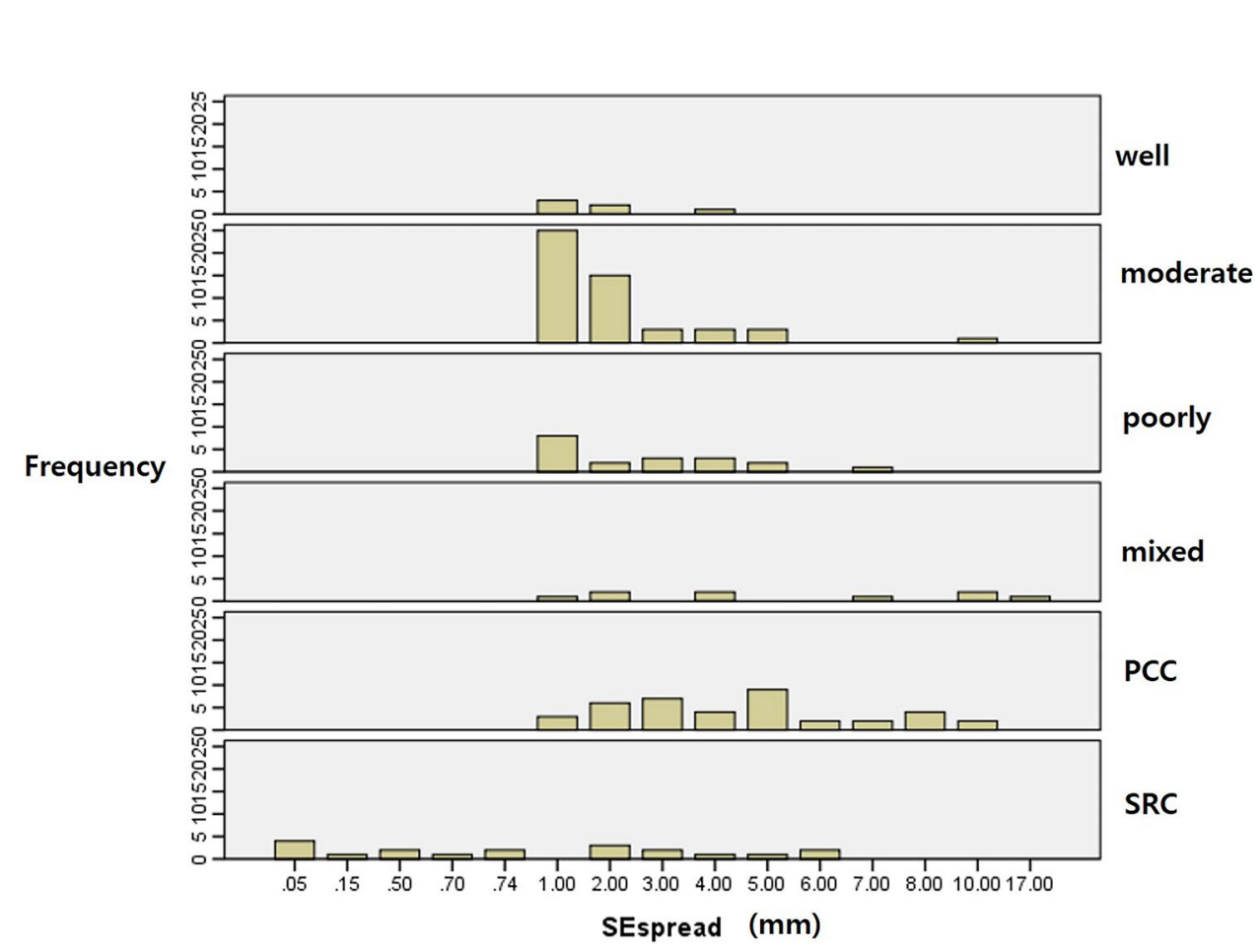

carcinoma, $S R C$ pure signet ring cell carcinoma
Fig. 2 Frequency and extent of subepithelial spreading for each histological type. The distance of subepithelial (SE) spread was significantly different according to histological type, with PCC and mixed type tending to have the greatest SE spreading distance (17 $\mathrm{mm}$ for mixed type). PCC: poorly cohesive carcinoma other than pure signet ring cell carcinoma, SRC: pure signet ring cell carcinoma
Therefore, it is important to determine an appropriate resection margin that balances oncological outcomes and other considerations. Endoscopists have no choice but rely on the extent of the visible lesion when determining the ESD margin. However, invisible lesion extension beneath the non-cancerous mucosa can cause LM positivity and non-curative resection [4]. We found that $35.1 \%$ of EGC patients had SE spread, which suggests that approximately one-third of these patients have a risk of LM positivity if the margin is based solely on endoscopic findings. A previous study suggested that non-curative resection is mainly related to positive deep margins for poorly differentiated cancer and positive lateral margins for SRC [7]. Surprisingly, the present study revealed that SE spread was common for moderately/poorly differentiated cancers, SRC, and PCC. If the extent of SE spread was within the range of endoscopic resection routinely set by endoscopists, LM positivity due to SE spread might not be an issue. The present study also revealed that, relative to PCC or SRC, the proportion of SE spreading to $>5 \mathrm{~mm}$ was not higher for moderately and poorly differentiated cancers (Fig. 3), which may explain the discrepancies between our findings and those of previous studies.

Kakushima et al. [4] reported that undifferentiated cancers had a higher proportion of LM positivity, relative to differentiated cancer, and the present study also revealed that SE spread was more common for undifferentiated cancers than for differentiated cancers. We have also previously reported that pure SRC had a high proportion of SE spread $(72.1 \%)$, which may be related to the specific growth pattern 
Fig. 3 Correlation between superficial lesion size and extent of subepithelial spread. The distance of subepithelial spread and superficial lesion size was not related (Pearson's correlation, $p=0.056$ )

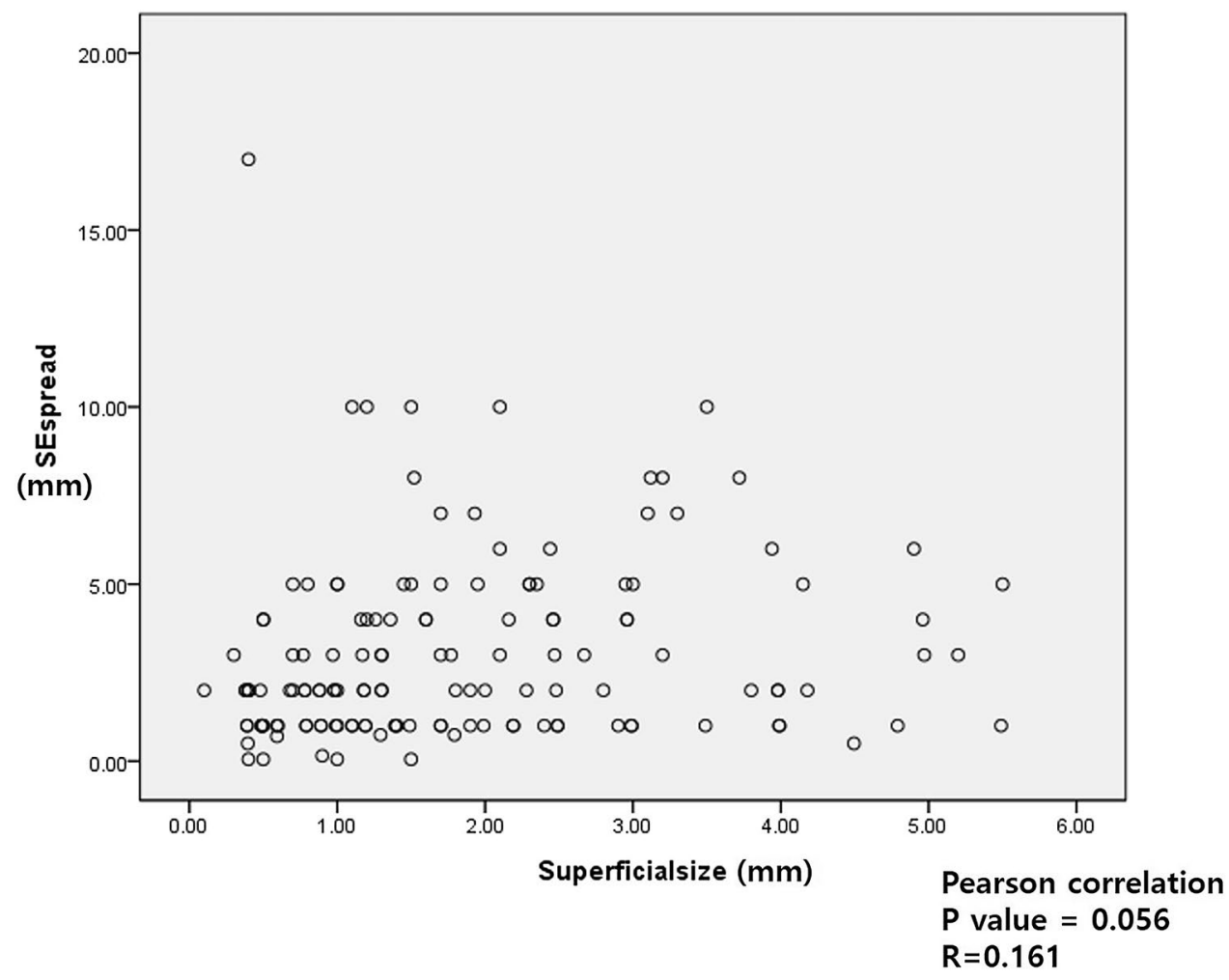

of tubule neck dysplasia $[5,8]$. As a presumed precursor to SRC, tubular neck dysplasia can spread vertically towards the foveolar surface and possibly deep towards the gastric glands [8]. Thus, SRC may have an intact epithelium, despite the presence of cancer cells in the lamina propria [9]. The 2010 WHO classification system categorized diffuse type poorly differentiated adenocarcinoma and SRC as "poorly cohesive carcinoma", although several studies have identified differences in biological behavior and clinical prognosis between SRC and diffuse type poorly differentiated adenocarcinoma [10-13]. Thus, we categorized poorly cohesive carcinoma as PCC (poorly cohesive carcinoma other than signet ring cell type) or SRC (pure signet ring cell carcinoma), based on the recently published consensus guideline, and we observed that PCC and SRC had a higher frequency of SE spread than differentiated cancers [6].

The present study revealed that the only independent risk factor for SE spread was histological type, and the mean distance of SE spread also varied according to histological type. Thus, a wider margin may be required for PCC and mixed type, relative to differentiated cancer. However, in contrast with our expectations, the distance of SE spread was relatively shorter for SRC (mean $1.9 \mathrm{~mm}$, maximum $6 \mathrm{~mm}$ ) than other histologic types (Table 4). Kim et al. [7] suggested that LM positivity was the main cause of incomplete resection in cases of SRC, however our results suggest that distance of SE spread in SRC might be within range of the endoscopic resection margins routinely set by endoscopists.

It is possible that LM positivity after ESD for SRC is related to the discolored tone of SRC, rather than SE spread, as the discolored tone makes it difficult to differentiate between the lesion and the surrounding mucosa. Furthermore, atrophic changes in the surrounding mucosa are a risk factor for underestimating the size of SRC [14]. Additional research is needed to evaluate this possibility.

The primary indication for ESD is M cancer, which prompted us to perform a subgroup analysis in patients who had negative submucosal and lymphatic invasion. In this subgroup analysis, histologic type was the only significant factor. The maximum SE spread distance in that subgroup analysis was $17 \mathrm{~mm}$, which was observed for mixed type. The maximal and mean value of SE spread distance in moderate differentiated cancer was 10 and $2 \mathrm{~mm}$. Our results have exceeded previous report which revealed the maximum extent of SE spread was $10 \mathrm{~mm}$ [3]. Therefore, a wide margin of $\geq 5 \mathrm{~mm}$ during ESD would likely be safe, 
Table 5 Univariate analysis of SE spread in patients who had negative submucosal and lymphatic invasion

\begin{tabular}{|c|c|c|c|}
\hline \multirow{2}{*}{$\begin{array}{l}\text { Characteristics } \\
\text { Gender }\end{array}$} & \multicolumn{2}{|c|}{ Presence of SE spread } & \multirow{2}{*}{$\frac{p \text { value }}{\mathbf{0 . 0 0 0}}$} \\
\hline & No & Yes & \\
\hline Male & 159 & 59 & \\
\hline Female & 46 & 33 & \\
\hline Histology & & & 0.000 \\
\hline Well & 77 & 6 & \\
\hline Moderate & 108 & 34 & \\
\hline Poorly & 9 & 12 & \\
\hline Mixed & 3 & 6 & \\
\hline PCC & 5 & 17 & \\
\hline $\mathrm{SRC}$ & 2 & 17 & \\
\hline Papillary & 1 & 0 & \\
\hline Location & & & 0.006 \\
\hline Cardia & 4 & 1 & \\
\hline Fundus & 0 & 1 & \\
\hline Upper body & 10 & 2 & \\
\hline Mid-body & 12 & 12 & \\
\hline Lower body & 35 & 24 & \\
\hline Angle & 21 & 16 & \\
\hline Antrum & 123 & 36 & \\
\hline Endoscopic appearance & & & $\mathbf{0 . 0 3 2}$ \\
\hline I & 7 & 4 & \\
\hline IIa & 47 & 8 & \\
\hline IIb & 31 & 21 & \\
\hline IIc & 106 & 49 & \\
\hline III & 14 & 10 & \\
\hline Elevation & & & 0.000 \\
\hline No & 114 & 72 & 0.000 \\
\hline Yes & 91 & 20 & \\
\hline Flat appearance & & & 0.005 \\
\hline No & 166 & 61 & \\
\hline Yes & 61 & 31 & \\
\hline Color change & & & 0.033 \\
\hline Redness & 120 & 47 & \\
\hline Discoloration & 57 & 21 & \\
\hline Mixed & 28 & 24 & \\
\hline
\end{tabular}

$\chi^{2}$ test: endoscopic appearance was classified according to the Paris classification

$S E$ subepithelial spread, $P C C$ poorly cohesive carcinoma other than pure signet ring cell carcinoma, $S R C$ pure signet ring cell carcinoma
Table 6 Logistic regression analysis of SE spread in patients who had negative submucosal and lymphatic invasion

\begin{tabular}{lrrll}
\hline Characteristics & $\beta$ coefficient & OR & $95 \%$ CI & $p$ value \\
\hline $\begin{array}{l}\text { Histology } \\
\text { (ref.=well) }\end{array}$ & & & & 0.000 \\
Moderate & 1.383 & 3.989 & $1.519-10.473$ & 0.005 \\
Poorly & 2.864 & 17.540 & $4.802-64.062$ & 0.000 \\
Mixed & 2.867 & 17.576 & $3.090-99.964$ & 0.001 \\
PCC & 3.743 & 42.213 & $10.208-174.564$ & 0.000 \\
SRC & 4.302 & 73.812 & $11.722-464.787$ & 0.000 \\
Papillary & -18.507 & 0.000 & 0.000 & 1.000 \\
\hline
\end{tabular}

All variants which are statistically significant in $\chi^{2}$ were included, and only histology was significant in logistic regression analysis

$S E$ subepithelial spread, ref. eference, $O R$ odds ratio, $C I$ confident interval, $P C C$ poorly cohesive carcinoma other than pure signet ring cell carcinoma, $S R C$ pure signet ring cell carcinoma

even for differentiated cancers. We often encounter cases with local recurrence after complete resection with enough safety margin [15]. Although there is limited evidence, local recurrence after complete resection may be associated with SE spread. Yun et al. have suggested that local recurrence after ESD was associated with safety margins of $<3 \mathrm{~mm}$, localization in the upper third of the stomach, and PCC and SRC histology [2]. These risk factors for local recurrence after ESD were similar to the risk factors for SE spread in the present study. Further researches will be needed to conclude this hypothesis.

The present study has several limitations. First, small single-center studies are prone to bias. Second, we were only able to evaluate a subset of cases that were treated at our center, as some slides had been used for other studies. Thus, it is possible that the range of SE spread might vary in a larger sample of cases. However, given the lack of data regarding SE spread, we believe that our results may help guide the optimization of ESD strategies.

In conclusion, we observed that SE spread was a common phenomenon in cases of early gastric cancer. A wider resection margin may be safe during endoscopic resection or surgery. 
Fig. 4 61-year-old female patient. a, b $0.4-\mathrm{cm}$-sized erythematous lesion with shallow ulcer was noted at lesser curvature side of mid-body. Surgical resection was done. c Blue dotted line represents surface invasion of cancer and yellow dotted line represents subepithelial spread of cancer. $\mathbf{d}$ Yellow circle represents $17 \mathrm{~mm}$ long subepithelial spread

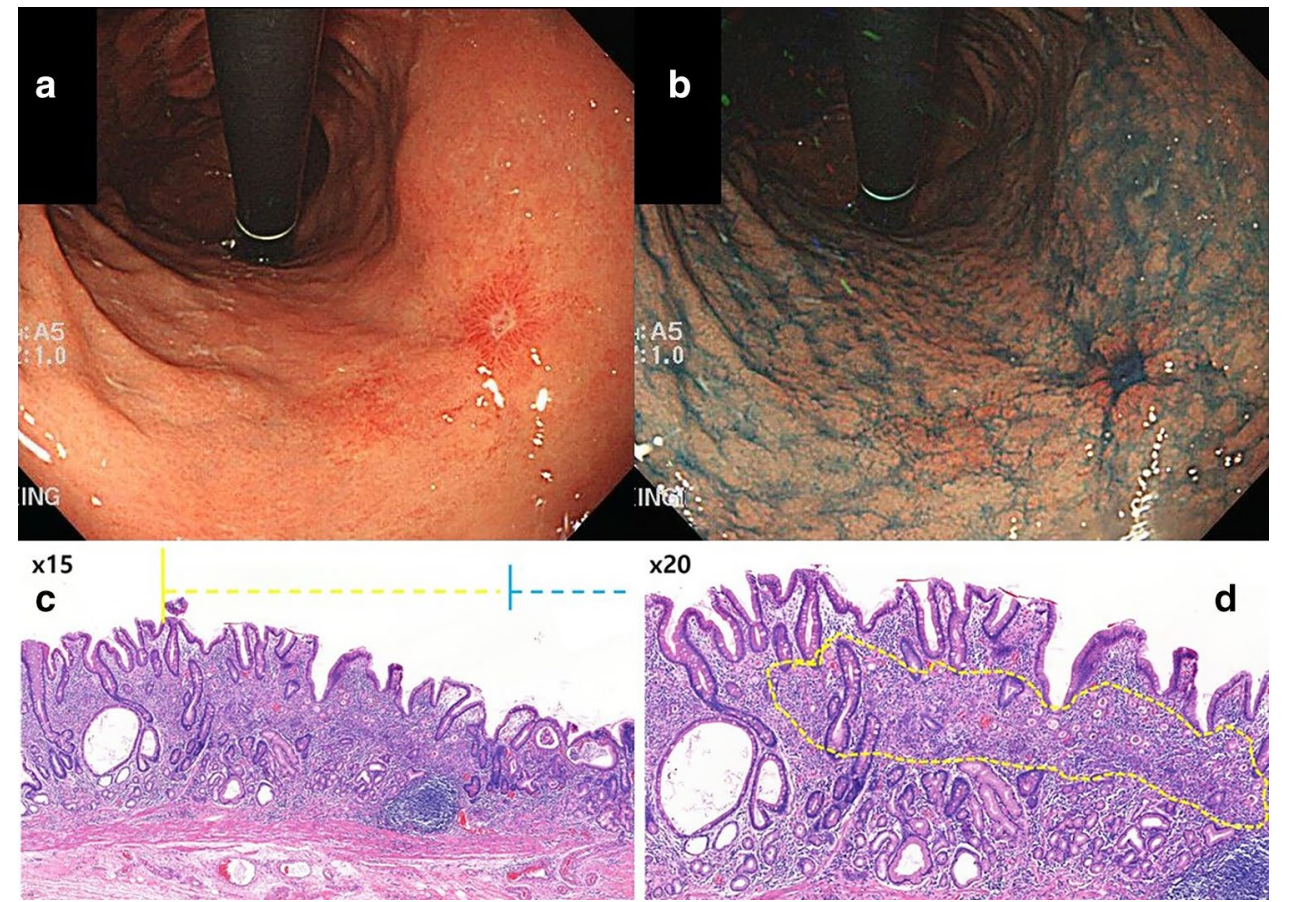

\begin{tabular}{llllcl}
\hline Histology & Mean $(\mathrm{mm})$ & SD & Minimum $(\mathrm{mm})$ & Maximum $(\mathrm{mm})$ & Number \\
\hline Well & 1.8333 & 1.16905 & 1.00 & 4.00 & 6 \\
Moderate & 2.0294 & 1.78352 & 1.00 & 10.00 & 34 \\
Poorly & 2.5000 & 1.97714 & 1.00 & 7.00 & 12 \\
Mixed & 7.8333 & 5.91326 & 1.00 & 17.00 & 6 \\
PCC & 4.2353 & 2.16591 & 1.00 & 10.00 & 17 \\
SRC & 2.0018 & 2.13231 & 0.05 & 6.00 & 17 \\
Total & 2.8590 & 2.77718 & 0.05 & 17.00 & 92 \\
\hline
\end{tabular}

Kruskal-wallis test: $p$ value $=0.000$

$S E$ subepithelial spread, $M$ cancer mucosal cancer, $S D$ standard deviation, $P C C$ poorly cohesive carcinoma other than pure signet ring cell carcinoma, $S R C$ pure signet ring cell carcinoma
Table 7 Difference of extent of SE spread between histologic types in patients who had negative submucosal and lymphatic invasion

\section{References}

Acknowledgements This work (research) was supported by Chungnam National University Hospital Research Fund, 2019.

\section{Compliance with ethical standard}

Conflict of Interest The authors declare that they have no conflict of interest.

Ethics approval All procedures complied with the ethical standards of the responsible committee on human experimentation (institutional and national) and with the Helsinki Declaration of 1964 and its later versions. This study's retrospective protocol was approved by the institutional review board of Chungnam National University Hospital (2020-04-159). The requirement for written consent was waived based on the retrospective design.
1. Francis G. Medicine: art or science? Lancet. 2020;395:24-5.

2. Yun GW, Kim JH, Lee YC, et al. What are the risk factors for residual tumor cells after endoscopic complete resection in gastric epithelial neoplasia? Surg Endosc. 2015;29:487-92.

3. Goto O, Fujimoto A, Shimoda M, et al. Estimation of subepithelial lateral extent in submucosal early gastric cancer: retrospective histological analysis. Gastric Cancer. 2015;18:810-6.

4. Kakushima N, Ono H, Tanaka M, Takizawa K, Yamaguchi Y, Matsubayashi H. Factors related to lateral margin positivity for cancers in gastric specimens of endoscopic submucosal dissection. Dig Endosc. 2011;23:227-32.

5. Lee YM, Kang SH, Kim JS, et al. Subepithelial spread of early gastric signet ring cell carcinoma: how far they can reach? Dig Dis. 2020;38(6):442-8.

6. Mariette C, Carneiro F, Grabsch HI, Post RS, Allum W, Manzoni Z. European chapter of international gastric cancer association 
consensus on the pathologic definition and classification of poorly cohesive gastric carcinoma. Gastric Cancer. 2019;22:1-9.

7. Kim JH, Lee YC, Kim HK, et al. Endoscopic resection for undifferentiated early gastric cancer. Gastrointest Endosc. 2009;69:e1-9.

8. Kumarasinghe MP, Lim TK, Ooi CJ, Luman W, Tan SY, Koh M. Tubule neck dysplasia: precursor lesion of signet ring cell carcinoma and the immunohistochemical profile. Pathology. 2006;38:468-71.

9. Kim HK, Kim JH, Lee YC, et al. Growth pattern of signet ring cell carcinoma of the stomach for endoscopic resection. Gut liver. 2015;9:720-6.

10. Lauwers GY, Carneiro F, Graham DY, Curado M-P, Franceschi S, Montgomery E, Tatematsu M, Hattori T. Gastric Carcinoma. In: Bosman FT, Carneiro F, Hruban RH, Theise ND, editors. WHO classification of tumours of the digestive system. 4th ed. Lyon: IARC Press; 2010. p. 48-58.

11. Kim YH, Kim JH, Kim HK, et al. Is the recent WHO classification for gastric cancer helpful for application to endoscopic resection? Gastric Cancer. 2016;19:869-75.
12. Kim YH, Park JH, Park CK, et al. Histologic purity of signet ring cell carcinoma is a favorable risk factor for lymph node metastasis in poorly cohesive, submucosa-invasive early gastric carcinoma. Gastric Cancer. 2017;20:583-90.

13. Kang SH, Kim JS, Moon HS, et al. Signet ring cell carcinoma of early gastric cancer, is endoscopic treatment really risky? Medicine. 2017;96:33.

14. Kim JS, Kang SH, Moon HS, et al. Accuracy of size measurement of early gastric signet ring cell carcinoma. Surg Endosc. 2020. https://doi.org/10.1007/s00464-020-07646-w.

15. Lee SH, Jung JH, Song JH, et al. Local recurrence of EGC after ESD. Clin Endosc. 2011;42:90-3.

Publisher's Note Springer Nature remains neutral with regard to jurisdictional claims in published maps and institutional affiliations. 